\title{
Dermatitis autoinmune por progesterona
}

\section{Autoimmune progesterone dermatitis}

Felicitas Peirano ${ }^{1}$, Jesica Radonich², Alejandro Sanz ${ }^{3}$, Miguel Ángel Mazzini ${ }^{4}$ y Florencia Galván ${ }^{5}$

\section{RESUMEN}

La dermatitis autoinmune por progesterona es un trastorno causado por una reacción de hipersensibilidad a la progesterona endógena o exógena. Afecta a mujeres en edad fértil y su frecuencia disminuye durante el embarazo. El cuadro clínico es variado y suele aparecer de forma cíclica, en relación directa con el ciclo menstrual. Los tratamientos incluyen la inhibición de la ovulación, el tratamiento desensibilizante $y$, en casos refractarios, se puede considerar, incluso, la ooforectomía bilateral con histerectomía.
Se presenta el caso de una paciente con dermatitis autoinmune por progesterona que realizó tratamiento desensibilizante con progesterona autóloga, con buena respuesta.

Palabras clave: dermatitis autoinmune por progesterona, tratamiento de desensibilización con progesterona autóloga.

Dermatol. Argent. 2020, 26 (4): 170-172

\section{ABSTRACT}

Autoimmune progesterone dermatitis is described as an hypersensitivity reaction to endogenous and/or exogenous progesterone. It affects women of childbearing age, decreasing its frequency during pregnancy. The clinical presentation is heterogeneous and skin lesions develop cyclically, in direct relation with the menstrual cycle. Among described treatments are the inhibition of the ovulation, desensitizing treatment and in refractory cases bilateral oophorectomy with hysterectomy can even be considered.
We present a case of autoimmune dermatitis caused by progesterone that was treated with desensitization with autologous progesterone with a good response.

Key words: autoimmune progesterone dermatitis, desensitization treatment with autologous progesterone.

Dermatol. Argent. 2020, 26 (4): 170-172
1 Médica Residente

2 Jefa de Residentes

3 Médico de Planta

${ }^{4}$ Jefe del Servicio

${ }^{5}$ Especialista en Dermatología

Servicio de Dermatología, Hospital Churruca-Visca, Ciudad

Autónoma de Buenos Aires, Argentina
Contacto del autor: Peirano Felicitas

E-mail: felicitaspeirano.fp@gmail.com

Fecha de trabajo recibido: $8 / 5 / 2020$

Fecha de trabajo aceptado: 19/11/2020

Conflicto de interés: los autores declaran que no existe conflicto de interés.

\section{CASO CLÍNICO}

Corresponde a una paciente de 37 años, con antecedentes de dislipidemia y migrańa, extabaquista, que realizó tratamiento con topiramato, amitriptilina y ácido valproico. Como antecedentes ginecológicos presentaba un parto eutócico a los 16 años y el uso de anticonceptivos orales durante 12 años, que suspendió 4 años antes. Consultó por una dermatosis de presentación cíclica, de 7 meses de evolución.

En el examen físico se observaban múltiples vesículas, agrupadas sobre una base eritematoedematosa, localizadas en ambas mejillas (de predominio derecho) y en la región supralabial, de 10 días de evolución, levemente pruriginosas (Fotos 1 y 2). La paciente refirió que el cuadro aparecía los días previos a la menstruación y se resolvía de forma espontánea unos días después. En el interrogatorio dirigido, señaló haber sido tratada con corticosteroides tópicos, pero estos no modificaron la evolución del cuadro. Negaba cambios en el uso de cosméticos, medicación o jabones en los últimos meses.

El estudio analítico incluyó un laboratorio completo con determinación de estrógenos y progesterona, que se encontraban dentro de los parámetros normales, y un citodiagnós- 
FOT0 1: Múltiples vesículas agrupadas sobre una base eritematoedematosa, localizadas en ambas mejillas, de predominio derecho y supralabial.

tico de Tzanck, que no evidenció células gigantes multinucleadas sugestivas de infección por el virus del herpes.

Se solicitó la interconsulta con el servicio de alergia e inmunología, donde realizaron intradermorreacciones con suero autólogo de la paciente en las fases lútea y folicular, que resultaron fuertemente positivas en la primera y negativas en la segunda. Asimismo, se efectuó una prueba de intradermorreacción con progesterona exógena con una solución de hidroxiprogesterona, que fue negativa. Así, con la clínica y la intradermorreacción positiva para progesterona endógena se arribó al diagnóstico de dermatitis autoinmune por progesterona.

Se indicó tratamiento desensibilizante con suero autólogo premenstrual, de forma subcutánea, semanal, en una concentración de $1 / 1.000$, que se aumentó de manera progresiva. La paciente presentó buena respuesta, sin reaparición de las lesiones tras 4 meses de tratamiento. La excelente respuesta al tratamiento instaurado confirmó el diagnóstico. No se realizó un estudio histopatológico, ya que suelen evidenciarse alteraciones inespecíficas.

\section{COMENTARIOS}

La dermatitis autoinmune por progesterona (DAP) es una patología de baja frecuencia, ya que se han publicado menos de 200 casos $^{1}$.

Suele presentarse con manifestaciones clínicas cíclicas, que se corresponden con la fase lútea del ciclo menstrual ${ }^{1}$. Se caracteriza por una reacción de hipersensibilidad a la progesterona endógena o exógena.
F0T0 2: Múltiples vesículas agrupadas sobre una base eritematoedematosa en una vista panorámica de la hemicara derecha.

Afecta a mujeres en edad fértil y su frecuencia es baja durante el embarazo, debido a la disminución de la respuesta inmunitaria y a la desensibilización que producen los niveles elevados de progesterona durante la gestación. Sin embargo, se han registrado casos excepcionales de empeoramiento durante el período gestante ${ }^{1-5}$.

Si bien no se cuenta con demasiada evidencia que respalde su carácter autoinmune, en los últimos años se comenzó a impulsar el término "hipersensibilidad a la progesterona" para describir esta condición. Hay nueva evidencia que sostiene que el cuadro clínico se desencadena luego de ocurrida una sensibilización a la progesterona ${ }^{1}$.

La fisiopatogenia es imprecisa, pero se han sugerido diversas teorías en cuanto a su desarrollo ${ }^{3}$. La progesterona es una hormona sexual producida principalmente por el cuerpo lúteo a lo largo de la vida de la mujer y por la placenta durante gran parte del embarazo ${ }^{1}$. Tanto la piel como la mucosa bucal tienen receptores de progesterona ${ }^{3}$. Se cree que algunas de las manifestaciones de este cuadro se producirían por reacciones de hipersensibilidad de tipo I o inmediata mediada por IgE, y otras a costa de un mecanismo de hipersensibilidad de tipo IV o retardada, mediado por la respuesta celular ${ }^{6,7}$.

Aunque no se conoce con certeza la forma en que se produce la sensibilización a la progesterona, se proponen diferentes hipótesis. Una de ellas plantea que la exposición a la progesterona exógena -durante los tratamientos anticonceptivos o de fertilidad- podría desencadenar, por medio de una reacción cruzada, el desarrollo de anticuerpos 
IgE contra la forma endógena de esa hormona ${ }^{1,8}$. En el caso presentado, la paciente había tenido una larga exposición a la progesterona exógena por el uso de anticonceptivos orales por un período mayor de 10 años, que provocó el cuadro clínico 4 años después de haberlos suspendido.

Sin embargo, hay casos de mujeres sin antecedentes de tratamientos hormonales previos que de todas formas padecen la enfermedad. El mecanismo propuesto para explicarlo es la posible reacción cruzada con esteroides (como puede ser la hidrocortisona) que, por tener una estructura similar a la progesterona, podrían desencadenar la producción de anticuerpos contra esta última ${ }^{1}$.

El espectro clínico de presentación es variado; sin embargo, en la mayoría de los casos las manifestaciones ocurren de manera cíclica alrededor de los 3 a 10 días previos al comienzo del ciclo menstrual. Esto se corresponde con la fase lútea, en la que se evidencia un aumento de los niveles de progesterona'. Si bien es menos frecuente durante el embarazo, el primer brote puede aparecer durante su transcurso ${ }^{7}$.

La urticaria y el prurito son las manifestaciones más frecuentes, pero la clínica es polimorfa y puede tener una distribución tanto localizada como generalizada. Se han publicado desde eccemas, exantemas maculopapulares o vesicoampollares, lesiones de tipo eritema multiforme, lesiones purpúricas, erupción fija por drogas, eritema anular centrífugo y angioedema hasta disnea, asma, tos e, incluso, anafilaxia, lo que puede retrasar el diagnóstico por años ${ }^{3,6,9,10}$. La mucosa también puede verse afectada; se describieron casos de estomatitis, aftas y prurito vulvovaginal ${ }^{1,5}$.

El diagnóstico, que se basa en la aparición cíclica característica de las manifestaciones clínicas, es de exclusión, porque la histología es inespecífica y las pruebas cutáneas no siempre son positivas ${ }^{3,7}$. La demostración de la sensibilización a la progesterona con el prick test, las pruebas

\section{BIBLIOGRAFÍA}

1. Foer $D$, Buchheit KM. Presentation and natural history of progestegen hypersensitivity. Ann Allergy Asthma Immunol 2019;122:156-159.

2. Orta DS, Abizuri Prado MF, Lecumberri Santamaría $B$, Merino $\mathrm{MB}$, et ál. Dermatitis autoinmune por progesterona o hipersensibilidad a la progesterona. Un nuevo caso con respuesta clínica a sulfona y revisión de la literatura. Med Cutan Iber Lat Am 2018;46:137-141.

3. Herzberg AJ, Strohmeyer CR, Cirillo-Hyland VA. Autoimmune progesterone dermatitis. J Am Acad Dermatol 1995;32:333-338.

4. Hill JL, Carr TF. latrogenic autoimmune progesterone dermatitis treated with a novel intramuscular progesterone desensitization protocol. J Allergy Clin Immunol Pract 2013;1:537-538.

5. Camões S, Sampaio J, Rocha J, Tiago P, et ál. Autoimmune progesterone dermatitis: case report of an unexpected treatment reaction. Australas J Dermatol 2017;58:e132-134.

6. Lee MK, Lee WY, Yong SJ, Shin KCH, et ál. A case of auto-inmmune progesterone dermatitis misdiagnosed as allergic contact intradérmicas inmediatas (30 minutos) y retardadas (24-48 horas) o la provocación intramuscular, vaginal u oral apoyan el diagnóstico ${ }^{3,7}$. Todas estas pruebas deben realizarse bajo control médico por la posibilidad de anafilaxia. Además, debe ponerse en evidencia la remisión de los síntomas tras la supresión de la progesterona al interrumpir la ovulación, luego de haber realizado técnicas de desensibilización ${ }^{7}$. En la paciente del caso, se evidenció una hipersensibilidad a la progesterona endógena y tuvo una excelente respuesta al tratamiento desensibilizante.

Dado que la clínica es tan variada, los diagnósticos diferenciales son múltiples y es necesario descartar enfermedades crónicas como lupus, acné, psoriasis, angioedema y dermatitis herpetiforme ${ }^{7}$.

El curso natural de la DAP es variable; se describieron casos de remisión espontánea, mientras que en algunas pacientes los síntomas se agravan con el tiempo ${ }^{7}$. Los corticosteroides tópicos o sistémicos y los antihistamínicos $\mathrm{H} 1$ pueden usarse para obtener un alivio sintomático ${ }^{9,10}$. En las pacientes que deben recibir altas dosis de progesterona porque están realizando tratamientos de fertilidad o en aquellas en quienes los síntomas se desencadenan durante el embarazo, se recomienda intentar la desensibilización a la progesterona mediante el uso de concentraciones ascendentes de forma oral o intramuscular ${ }^{11}$.

Los anticonceptivos orales que contienen estrógeno y progestágeno son el abordaje de primera línea9. La respuesta al tratamiento se da en los casos en los que no hay reactividad cruzada con la progesterona exógena ${ }^{12}$. Las terapias hormonales con análogos de la hormona liberadora de gonadotropinas, tamoxifeno o danazol podrían ser más adecuadas en las pacientes perimenopáusicas y su uso debe limitarse a un período de 6 meses $^{7}$.

En todas las pacientes los síntomas se resuelven en su totalidad con la ooforectomía, pero se la reserva para los casos graves y para las mujeres con paridad cumplida ${ }^{10}$.

dermatitis. Allergy Asthma Immunol Res 2011;3:141-144.

7. Nguyen T, Razzaque Ahmed A. Autoimmune progesterone dermatitis: update and insights. Autoimmun Rev 2016;15:191-197.

8. Solomon $M$, Itsekson AM, Lev-Sagie A. Autoimmune progesterone dermatitis. Curr Derm Rep 2013;2:258-263.

9. Baptist AP, Baldwin JL. Autoimmune progesterona dermatitis in a patient with endometriosis: case report and review of the literature. Clin Mol Allergy 2004;2:10.

10. Snyder JL, Krishnaswamy G. Autoimmune progesterone dermatitis and its manifestation as anaphylaxis: a case report and literature review. Ann Allergy Asthma Immunol 2003;90:469-477.

11. Foer D, Buchheit KM, Gargiulo AR, Lynch DM, et ál. Progestogen hypersensitivity in 24 cases: diagnosis, management, and proposed renaming and classification. J Allergy Clin Immunol Pract 2016;4:723-729.

12. Frieder J, Younus M. Autoimmune progesterone dermatitis with delayed intradermal skin reaction: a case report. Ann Allergy Asthma Immunol 2016;117:438-439. 